\title{
Review of Handbook of Physics in Medicine and Biology, edited by Robert Splinter
}

Ivan A Dotsinsky

Correspondence: iadoc@bas.bg Center of Biomedical Engineering, Bulgarian Academy of Sciences, 105, Acad. G. Bonchev Str., 1113 Sofia, Bulgaria

\section{Book details \\ Robert Splinter (Ed): \\ Handbook of Physics in Medicine and Biology. CRC Press, Taylor \& Francis Group, 2010, ISBN 978-1-4200-7524-3, 548 pages}

I have always been astonished whenever I open an encyclopedic book, imagining the effort it took to review so many closely related but actually different scientific contributions, and at the same time to provide the detailed interpretations needed to satisfy the experts in the field. I felt the same respect when I picked up the Handbook of Physics in Medicine and Biology, which was edited by Robert Splinter and published by the Taylor \& Francis Group.

I quickly read over the entire book, then read parts of it in detail. My deep impression is this is an excellent work by a highly competent team. The book chapters follow logically from the properties of the cell membrane through sensors and electroreception, biomechanics and fluid dynamics to the recording of bioelectrical signals, bioelectric impedance analysis, X-ray and computed tomography, magnetic resonance imaging, nuclear medicine, ultrasonic and thermographic imaging. I am less familiar with some of the microscopy and optical techniques as well as the lab-on-a-chip and the biophysics of regenerative medicine, which covers a breadth material that can hardly be learned by any single person. Therefore, I will confine my comments to the book's presentation of techniques that I have used in the last decades, keeping in mind the impossibility of achieving a perfect balance between providing foundations of the subjects for students and advanced discussion of the topics for professionals.

The Handbook's discussion of fundamental properties of the cell membrane is well organized. However, I missed some applied topics of interest to bioengineers such as electrochemotherapy - a modern method of treating tumors that uses short but intense pulses of electric fields to permeabilize (electroporate) cell membranes to enhance the administration of cytotoxic drugs.

The Handbook also provides methodical, perspicuous and very useful discussions of action potential transmission and volume conduction. This is true also for the chapter devoted to the physics of reception which gives profound information about the different senses: taste, smell, touch, pain, as well as hearing and vision. I was particularly interested in a short chapter dealing with biomedical engineering contributions to medical decision making. The progress in electronics, computer science and biomedical technologies has created higher expectances of enhanced technical contributions to

(C) 2010 Dotsinsky; licensee BioMed Central Ltd. This is an Open Access article distributed under the terms of the Creative Commons Attribution License (http://creativecommons.org/licenses/by/2.0), which permits unrestricted use, distribution, and reproduction in any medium, provided the original work is properly cited. 
medical decision making. In my opinion, these expectations are somewhat exaggerated and reflect overenthusiasm by their proponents. In fact, most expert systems for medical decision making are able to generate diagnostic conclusions more accurately than those produced by ordinary clinicians. However, a great diagnostician can sometimes factor in surprising but not statistically relevant data that might be a key consideration for an accurate decision. This is due to the specific thought processes of skilled physicians, who do not go in sequence through an algorithm as does a computer program. By the way, the mathematical requirements of Bayes' theorem (a fundamental theorem that governs the accuracy of medical decision making) for independence and invariability of the events as well as the presence of whole-group marginal events are not discussed in this chapter.

The Handbook offers a compressed discussion of the complex topic of biomechanics that introduces the basic information of the three muscle types. It includes a very interesting paragraph, which describes the technology of micro electro-mechanical systems as they are used in artificial muscles.

Also included is necessary information about the functional anatomy of the heart, the cardiac cycle (systole and diastole) and the mechanics of the cardiovascular system. The measurement of arterial blood pressure is given in terms of Korotkoff's sounds only. A parallel description of blood pressure measurement using the oscillatory principle would be very valuable, since both techniques are almost equally used in practice.

The chapters on fluid dynamics of the cardiovascular system and the discussion of stroke volume (SV) and the derivative cardiac output provoked me to share some considerations. As it is well known, there are a variety of methods to measure SV, e.g. the dye dilution, Doppler ultrasound, sphygmomanometry and tonometry etc, as well as some invasive methods. No one of these is recognized as the "gold standard" for accuracy, and each has its own supporters. Long ago my colleagues and I used impedance cardiography for monitoring the SV in the intensive care units in Bulgaria. The onset of the systolic wave, its peak and the most negative deflection corresponding to the closing of the aortic valve were detected from the time rate of change of the impedance signal. They were used to derive the SV according to the Korotkoff's formula but not before the fiducial points shown on the screen were approved by the operator. Further, the method we employed allowed for the possibility of correcting coefficients for adjusting the obtained data, virtually corresponding to another method if it has been applied once at the beginning to the monitored patient.

The Handbook includes a very nice presentation of the anatomy and physics of respiration, including the measurement of lung volumes, gas exchange and transport in the blood. There is also a superb review of ventilation. I missed a discussion of the problem of estimating the optimal moment of transition from mandatory to spontaneous breathing. Perhaps the answer to this problem is beyond the scope of a handbook.

As expected, much space is devoted to the recording of bioelectric signals. I am happy with the chapter dealing with the electrocardiogram (ECG), which includes the entire sequence of topics, from placement of electrodes on the body, lead formation of the acquired signals, description of the waves' origin, wave shape and duration, intervals and segments, continuing on to a description of abnormalities of the ECG in several cardiac diseases. It appears to me that a little more information on the rhythm 
disorders would have been suitable; only one figure shows the shape of ectopic beats within a bigeminic epoch. I would have wanted a few words devoted to the disturbances accompanying the ECG signal acquisition - power line interference, drift, tremor and other artefacts, which may hamper or even distort the morphological interpretation of ECG waveforms.

The basic concepts and applications of electroencephalography (EEG) are also well covered. The authors of that chapter have emphasized evoked and event-related brain potentials together with the signal averaging techniques necessary for their correct interpretation. The EEG is routinely analyzed by frequency analysis. The chapter would benefit by including a discussion of Fourier analysis with a few sentences about some inconveniences of that approach, namely the loss of the time parameter and the energy leakage in the spectrum due to incorrect length of the selected epoch. These disadvantages can be overcome using the method of the first zero derivatives.

I also appreciated the presentation of X-ray instrumentation, computed tomography (CT), magnetic resonance imaging (MRI) and ultrasound imaging, which covers a good part of the biomedical imaging discussed in the Handbook; other highly useful technologies presented are positron emission tomography, thermography and different types of microscopy. The chapter on X-ray imaging lacks the definitions of some very important parameters of the X-ray image - brightness, contrast and unsharpness. The central slice theorem is well presented, but it would have been helpful to have enlarged the discussion of algorithm for CT image reconstruction e.g. back projection, iterative and analytic algorithm. The reconstruction of the MRI image is totally ignored.

In conclusion, the authors' team and the Taylor \& Francis Group should be congratulated for this excellent handbook. My minor criticisms represent a particular look at the material and are not intended to disparage the overall high worth of the book. I suggest to readers of BioMedical Engineering OnLine: keep this book close at hand and do not hesitate to use it frequently.

\author{
Submit your next manuscript to BioMed Central \\ and take full advantage of: \\ - Convenient online submission \\ - Thorough peer review \\ - No space constraints or color figure charges \\ - Immediate publication on acceptance \\ - Inclusion in PubMed, CAS, Scopus and Google Scholar \\ - Research which is freely available for redistribution

